United Nations Educational Scientific and Cultural Organization

and

International Atomic Energy Agency

THE ABDUS SALAM INTERNATIONAL CENTRE FOR THEORETICAL PHYSICS

\title{
ON RANDOM APPROXIMATIONS \\ AND RANDOM FIXED POINT THEOREMS \\ FOR 1-SET-CONTRACTIVE RANDOM MAPS
}

\author{
Naseer Shahzad \\ Department of Mathematics, Quaid-i-Azam University, Islamabad, Pakistan ${ }^{1}$ \\ and \\ The Abdus Salam International Centre for Theoretical Physics, Trieste, Italy \\ and \\ Saadia Latif \\ Department of Mathematics, Quaid-i-Azam University, Islamabad, Pakistan.
}

\begin{abstract}
The validity of Fan's best approximation theorem for a continuous 1-set-contractive random map defined on a separable closed ball in a Banach space is established. As applications, some random approximation and random fixed point theorems are derived.
\end{abstract}

MIRAMARE - TRIESTE

July 1998

\footnotetext{
${ }^{1}$ Permanent address.
} 


\section{INTRODUCTION}

In 1969, Fan [3] proved a very interesting result that combined fixed point theory with the study of proximity maps. Its normed space version [3, Theorem 2] is stated as follows:

Let $K$ be a non-empty compact convex set in a normed space $X$. For any continuous map $T$ from $K$ into $X$, there exists a point $x$ in $K$ such that

$$
\|x-T(x)\|=d(T(x), K) .
$$

Fan's theorem has non-trivial implications in the areas of nonlinear analysis, approximation theory, minimax theorems, and to the burgeoing field of the theory of games. The interest in these problems was revived by the article of Lin [8]. Since then there has appeared a number of interesting nonstochastic analogues of Fan's theorem (see, for example, the work of Fan [4], Ha [5], Lin [9], Lin and Yen [12], Singh and Watson [22], etc.). Afterwards, Sehgal and Waters [20], Sehgal and Singh [19], Papageorgiou [16, 17], Lin [10, 11], Beg and Shahzad [1, 2], Tan and Yuan [23, 24], and Shahzad [21] studied different random versions of the above theorem. Lin [10] considered a continuous condensing map defined on a separable closed ball in a Banach space. Recently, Liu [13] extended Lin's result to more general 1-set-contractive random operators defined on a separable weakly compact closed ball in a Banach space. As applications, he also obtained some random fixed point theorems. The aim of this paper is to give different stochastic versions of Fan's theorem and to derive, as applications, new random fixed point theorems for non-self maps. Instead of considering a separable weakly compact ball as in Liu [13], we consider a separable closed ball in a Banach space and establish the validity of Fan's theorem for 1-set contractive random operators defined on it.

\section{Preliminaries}

Throughout this paper, $(\Omega, \Sigma)$ denotes a measurable space with $\Sigma$ a sigma algebra of subsets of $\Omega$. Let $S$ be a non-empty subset of a Banach space $X$. We denote by $2^{S}$ the family of all subsets of $S$ and by $C(S)$ the family of all non-empty closed subsets of $S$. A mapping $G: \Omega \longrightarrow 2^{S} \backslash\{\phi\}$ is called measurable if, for each open subset $U$ of $S, G^{-1}(U)=\{\omega \in \Omega$ : $G(\omega) \cap U \neq \phi\} \in \Sigma$. A mapping $\xi: \Omega \longrightarrow S$ is said to be a measurable selector of measurable mapping $G: \Omega \longrightarrow 2^{S} \backslash\{\phi\}$ if $\xi$ is measurable and $\xi(\omega) \in G(\omega)$ for each $\omega \in \Omega$. A mapping $T: \Omega \times S \longrightarrow X$ is called a random operator if, for any fixed $x \in S$, the map $T(., x): \Omega \longrightarrow X$ is measurable. A mapping $\xi: \Omega \longrightarrow S$ is said to be a random fixed point of a random operator $T: \Omega \times S \longrightarrow X$ if $\xi(\omega)=T(\omega, \xi(\omega))$ for each $\omega \in \Omega$. Let $A$ be a non-empty bounded subset of $S$, and let $\alpha(A)=\inf \{c>0: A$ can be covered by a finite number of sets of diameter $\leq c\}$. A mapping $T: S \longrightarrow X$ is called (1) $k$-set-contractive if, for each bounded subset $A$ of $S, \alpha(T(A)) \leq k \alpha(A)$, where $k \geq 0$; (2) condensing if, for each bounded subset $A$ of $S$ with $\alpha(A)>0, \alpha(T(A))<\alpha(A)$; (3) contraction if $\|T(x)-T(y)\| \leq k\|x-y\|$ for each $x, y \in S$, where $0 \leq k<1$; (4) nonexpansive if $\|T(x)-T(y)\| \leq\|x-y\|$ for each $x, y \in S$; (5) generalized 
contraction if, for each $x \in S$, there exists a number $k(x)<1$ with $\|T(x)-T(y)\| \leq k(x)\|x-y\|$ for each $y \in S$; $(6)$ completely continuous if it maps weakly convergent sequences into strongly convergent sequences; (7) compact if $\overline{T(A)}$ is compact whenever $A \subset X$ is bounded; (8) uniformly strictly contractive on $\mathrm{S}$ relative to $X$ if the map $T: X \longrightarrow X$ has the property that, for each $x \in X$, there exists a number $k(x)<1$ such that $\|T(x)-T(y)\| \leq k(x)\|x-y\|$ for each $y \in S$; (9) LANE ( locally almost nonexpansive) if, for each $x \in S$ and $\epsilon>0$, there exists a weak neighborhood $N_{x}$ of $x$ in $S$ (depending also on $\epsilon$ ) such that $\|T(u)-T(v)\| \leq\|u-v\|+\epsilon$ for each $u, v \in N_{x}$.

Let $S$ be a non-empty closed bounded subset of a Banach space $X$ and $T: S \longrightarrow X$ a continuous map. (a) Suppose there exists a continuous map $V: S \times S \longrightarrow X$ such that $T(x)=V(x, x)$ for $x \in S$. Then (i) $T$ is of strictly semicontractive type if, for each $x \in S$, $V(., x)$ is a contraction and the map $x \longrightarrow V(., x)$ of $S$ into the space of continuous mappings of $S$ into $X$ with the uniform metric is compact; (ii) $T$ is of weakly semicontractive type if, for each $x \in S, V(., x)$ is a nonexpansive map of $S$ into $X$ and $x \longrightarrow V(., x)$ of $S$ into the space of continuous mappings of $S$ into $X$ is compact. (b) Suppose there exists a mapping $V: X \times S \longrightarrow X$ such that $T(x)=V(x, x)$ for $x \in S$. Then $T$ is of strongly semicontractive type relative to $X$ if, for each $x \in S$, the mapping $V(., x)$ is uniformly strictly contractive on $S$ relative to $X$ and $V(x,$.$) is completely continuous from S$ to $X$, uniformly for $x \in S$. We remark that all of these mappings are 1-set-contractive. For more details of such mappings, see Kirk [6] and Petryshyn [18].

A mapping $T: S \longrightarrow X$ is called hemicompact if each sequence $\left\{x_{n}\right\}$ in $S$ has a convergent subsequence whenever $\left\|x_{n}-T\left(x_{n}\right)\right\| \longrightarrow 0$ as $n \longrightarrow \infty$. A mapping $T: S \longrightarrow X$ is said to satisfy the condition $(A)$ if for any sequence $\left\{x_{n}\right\}$ in $S$ and $D \in C(S)$ such that $d\left(x_{n}, D\right) \longrightarrow 0$ and $\left\|x_{n}-T\left(x_{n}\right)\right\| \longrightarrow 0$ (equivalently $x_{n}-T\left(x_{n}\right) \longrightarrow 0$ ) as $n \longrightarrow \infty$, there exists $x_{0} \in D$ with $x_{0}-T\left(x_{0}\right)=0$. It is easy to see that every continuous hemicompact map satisfies the condition $(A)$. We also remark that the condition $(A)$ is always true for continuous condensing mappings. A random operator $T: \Omega \times S \longrightarrow X$ is said to be continuous (1-set-contractive, condensing, compact, generalized contraction, LANE, of semicontractive type, etc.) if the map $T(\omega,):. S \longrightarrow X$ is so for each $\omega \in \Omega$.

\section{RANDOM APPROXIMATIONS}

We start with the following simple result.

Lemma 3.1. Let $S$ be a non-empty separable closed bounded subset of a Banach space $X$ and $T: \Omega \times S \longrightarrow S$ either a continuous 1-set-contractive or a continuous 1-ball-contractive random operator for which the condition $(A)$ holds. Then $T$ has a random fixed point. 
Proof. Define a mapping $G: \Omega \longrightarrow 2^{X}$ by $G(\omega)=\{x \in S: x=T(\omega, x)\}$. By Petryshyn[18, Theorem 1], $G(\omega)$ is non-empty for each $\omega \in \Omega$. For each non-empty closed subset $D$ of $S$, let

$$
L(D)=\bigcap_{n=1}^{\infty} \bigcup_{x \in D_{n}}\left\{\omega \in \Omega: d(x, T(\omega, x))<\frac{2}{n}\right\},
$$

where $D_{n}=\left\{x \in S: d(x, D)<\frac{1}{n}\right\}$ and $d(x, D)=\inf \{d(x, y): y \in D\}$. We claim that $G^{-1}(D)=L(D)$. Indeed, it is easy to see that $G^{-1}(D) \subset L(D)$. Conversely, if $\omega \in L(D)$, then, for each $n$, there exists $x_{n}$ such that $d\left(x_{n}, D\right)<\frac{1}{n}$ and $d\left(x_{n}, T\left(\omega, x_{n}\right)\right)<\frac{2}{n}$. It further implies that $d\left(x_{n}, D\right) \longrightarrow 0$ and $d\left(x_{n}, T\left(\omega, x_{n}\right)\right) \longrightarrow 0$ as $n \longrightarrow \infty$. An application of the condition (A) yields $x_{0}-T\left(\omega, x_{0}\right)=0$ for some $x_{0} \in D$. Hence $\omega \in G^{-1}(D)$ and so $G^{-1}(D)=L(D)$. Also $G^{-1}(D)=L(D) \in \Sigma$ and $G$ is measurable. Since $G$ has closed values, it follows from the Kuratowski and Ryll-Nardzewski selection theorem [7] that there exists a measurable mapping $\xi: \Omega \longrightarrow S$ such that $\xi(\omega) \in G(\omega)$ for each $\omega \in \Omega$. It is obvious that $\xi$ is a random fixed point of $T$.

Using the arguments analogous to those in [13] we now establish the following lemma which plays an important role in the sequel.

Lemma 3.2. Let $S$ be a closed ball with center at the origin and radius $r$ in a Banach space $X$. If $T: S \longrightarrow X$ is a continuous 1-set-contractive mapping satisfying the condition $(A)$, then the mapping $H \equiv R T: S \longrightarrow S$ also satisfies the condition $(A)$, where $R: X \longrightarrow S$ is defined by

$$
R(x)= \begin{cases}x & \text { if }\|x\| \leq r \\ \frac{r x}{\|x\|} & \text { if }\|x\| \geq r .\end{cases}
$$

Proof. Let $\left\{x_{n}\right\}$ be any sequence in $S$ and $D \in C(S)$ such that $d\left(x_{n}, D\right) \longrightarrow 0,\left\|x_{n}-H\left(x_{n}\right)\right\| \longrightarrow$ 0 (that is, $x_{n}-H\left(x_{n}\right) \longrightarrow 0$ ) as $n \longrightarrow \infty$.

If there exists a subsequence $\left\{x_{m}\right\}$ of $\left\{x_{n}\right\}$ such that $\left\|T\left(x_{m}\right)\right\| \leq r$ for all $m$, then $H\left(x_{m}\right)=$ $T\left(x_{m}\right)$. Hence there exists $x_{0} \in D$ such that $x_{0}-T\left(x_{0}\right)=0$. But $x_{0}-H\left(x_{0}\right)=x_{0}-R\left(T\left(x_{0}\right)\right)=$ $x_{0}-T\left(x_{0}\right)=0$.

If there exists an integer $N$ such that $\left\|T\left(x_{n}\right)\right\|>r$ for $n \geq N$, then $H\left(x_{n}\right)=\frac{r T\left(x_{n}\right)}{\left\|T\left(x_{n}\right)\right\|}$. Since $\left\{x_{n}\right\}$ is bounded and $T$ is 1-set-contractive, $\left\{\left\|T\left(x_{n}\right)\right\|\right\}$ is bounded. And so there exist a subsequence $\left\{\left\|T\left(x_{k}\right)\right\|\right\}$ of $\left\{\left\|T\left(x_{n}\right)\right\|\right\}$ and a real number $L>0$ such that $\left\|T\left(x_{n}\right)\right\| \rightarrow L$ an $k \longrightarrow \infty$. Since $\left\|T\left(x_{k}\right)\right\|>r$ for all $k, L \geq r$. Thus $x_{k}-\frac{r}{L} T\left(x_{k}\right)=\left(x_{k}-\frac{r T\left(x_{k}\right)}{\left\|T\left(x_{k}\right)\right\|}\right)+\left(\frac{r T\left(x_{k}\right)}{\left\|T\left(x_{k}\right)\right\|}-\right.$ $\left.\frac{r}{L} T\left(x_{k}\right)\right)=\left(x_{k}-H\left(x_{k}\right)\right)+\left(\frac{1}{\left\|T\left(x_{k}\right)\right\|}-\frac{1}{L}\right) r T\left(x_{k}\right) \longrightarrow 0$ as $k \longrightarrow \infty$. If $L=r$, then $x_{k}-T\left(x_{k}\right) \longrightarrow 0$ as $k \longrightarrow \infty$ (in other words $\left\|x_{k}-T\left(x_{k}\right)\right\| \longrightarrow 0$ as $k \longrightarrow \infty$ ). An application of the condition $(A)$ yields $x_{0}-T\left(x_{0}\right)=0$ for some $x_{0} \in D$. Therefore $x_{0}-H\left(x_{0}\right)=x_{0}-R\left(T\left(x_{0}\right)\right)=x_{0}-T\left(x_{0}\right)=0$. If $L>r$, then $\left(\frac{r}{L}\right) T$ is a $\frac{r}{L}$-set-contractive mapping with $\frac{r}{L}<1$. Set $y_{k}=x_{k}-\frac{r}{L} T\left(x_{k}\right)$. Then $\alpha\left(\left\{y_{k}\right\}\right)=0$. Since $x_{k}=y_{k}+\frac{r}{L} T\left(x_{k}\right)$, it follows that $\alpha\left(\left\{x_{k}\right\}\right)=0$ and so there exists a subsequence $\left\{x_{l}\right\}$ of $\left\{x_{k}\right\}$ such that $x_{l} \longrightarrow x_{0}$. It further implies by the continuity of $T$ that $x_{0} \in D$ and $x_{0}-\frac{r}{L} T\left(x_{0}\right)=0$. Again, since $\left\|T\left(x_{l}\right)\right\| \longrightarrow L$ as $l \longrightarrow \infty$, we have $\left\|T\left(x_{0}\right)\right\|=L>r$. Thus $x_{0}-H\left(x_{0}\right)=x_{0}-\frac{r T\left(x_{0}\right)}{\left\|T\left(x_{0}\right)\right\|}=x_{0}-\frac{r}{L} T\left(x_{0}\right)=0$. Hence $H$ satisfies the condition $(A)$. 
Theorem 3.3. Let $S$ be a separable closed ball with center at the origin and radius $r$ in a Banach space $X$ and $T: \Omega \times S \longrightarrow X$ a continuous 1-set-contractive random operator for which the condition $(A)$ holds. Then there exists a measurable map $\xi: \Omega \longrightarrow S$ such that

$$
\|\xi(\omega)-T(\omega, \xi(\omega))\|=d(T(\omega, \xi(\omega)), S)
$$

for each $\omega \in \Omega$.

Proof. Let $R: X \longrightarrow S$ be a map defined by

$$
R(x)= \begin{cases}x & \text { if }\|x\| \leq r \\ \frac{r x}{\|x\|} & \text { if }\|x\| \geq r\end{cases}
$$

By Nussbaum[15, Corollary 1], $R$ is a continuous 1-set-contractive map. Define $F: \Omega \times S \longrightarrow S$ by $F(\omega, x)=R(T(\omega, x))$. Then $F$ is a 1-set-contractive random operator. By Lemma 3.2, $F(\omega,$.$) satisfies the condition (A)$ for each $\omega \in \Omega$. Therefore, by Lemma $3.1, F$ has a random fixed point $\xi: \Omega \longrightarrow S$. It remains to show that this measurable map $\xi$ satisfies the desired property. For any $\omega \in \Omega$,

$$
\begin{gathered}
\|\xi(\omega)-T(\omega, \xi(\omega))\|=\|F(\omega, \xi(\omega))-T(\omega, \xi(\omega))\| \\
=\|R(T(\omega, \xi(\omega)))-T(\omega, \xi(\omega))\| \\
= \begin{cases}\|T(\omega, \xi(\omega))-T(\omega, \xi(\omega))\| & \text { if }\|T(\omega, \xi(\omega))\| \leq r \\
\left\|\frac{r T(\omega, \xi(\omega))}{\|T(\omega, \xi(\omega))\|}-T(\omega, \xi(\omega))\right\|=\|T(\omega, \xi(\omega))\|-r & \text { if }\|T(\omega, \xi(\omega))\| \geq r .\end{cases}
\end{gathered}
$$

Thus, for any $x \in S$, we obtain

$$
\begin{gathered}
\|\xi(\omega)-T(\omega, \xi(\omega))\| \leq\|T(\omega, \xi(\omega))\|-r \\
\leq\|T(\omega, \xi(\omega))\|-\|x\| \leq\|T(\omega, \xi(\omega))-x\| .
\end{gathered}
$$

Hence

$$
\|\xi(\omega)-T(\omega, \xi(\omega))\|=d(T(\omega, \xi(\omega)), S)
$$

for each $\omega \in \Omega$.

Remark 3.4. As in the proof of [13, Theorem 2.1], the measurable map $\xi: \Omega \rightarrow S$ of Theoem 3.3 has the following properties:

For any $\omega \in \Omega$, if $\|T(\omega, \xi(\omega))\| \geq r$, then $\xi(\omega) \in S_{r}=\{x \in X:\|x\|=r\}$ and

$$
\xi(\omega)=\frac{r T(\omega, \xi(\omega))}{\|T(\omega, \xi(\omega))\|},\|\xi(\omega)-T(\omega, \xi(\omega))\|=\|T(\omega, \xi(\omega))\|-r>0 ;
$$

if $\|T(\omega, \xi(\omega))\| \leq r$, then $\xi$ is the random fixed point of $T$.

Corollary 3.5. Let $S$ be a separable closed ball with center at the origin and radius $r$ in a Banach space $X$ and $T: \Omega \times S \longrightarrow X$ a continuous condensing random operator. Then there exists a measurable map $\xi: \Omega \longrightarrow S$ such that 


$$
\|\xi(\omega)-T(\omega, \xi(\omega))\|=d(T(\omega, \xi(\omega)), S)
$$

for each $\omega \in \Omega$.

Proof. Since every continuous condensing map satisfies the condition $(A)$, this corollary follows immediately from Theorem 3.3.

Remark 3.6. Corollary 3.5 slightly generalizes Lin[10, Theorem1].

Corollary 3.7. Let $S$ be a separable closed ball with center at the origin and radius $r$ in a Banach space $X, B: \Omega \times S \longrightarrow X$ a random contraction, and $C: \Omega \times S \longrightarrow X$ a continuous compact random operator. If $T=B+C$, then there exists a measurable map $\xi: \Omega \longrightarrow S$ such that

$$
\|\xi(\omega)-T(\omega, \xi(\omega))\|=d(T(\omega, \xi(\omega)), S)
$$

for each $\omega \in \Omega$.

Proof. Since $B: \Omega \times S \longrightarrow X$ is a random contraction and $C: \Omega \times S \longrightarrow X$ is a continuous compact random operator, $T=B+C: \Omega \times S \longrightarrow X$ is a continuous condensing random operator and so satisfies the condition $(A)$. The corollary just follows from Theorem 3.3.

Theorem 3.8. Let $S$ be a separable closed ball with center at the origin and radius $r$ in a reflexive Banach space $X, B: \Omega \times S \longrightarrow X$ a uniformly strictly contractive random operator on $S$ relative to $X$, and $C: \Omega \times S \longrightarrow X$ a continuous compact random operator. If $T=B+C$, then there exists a measurable map $\xi: \Omega \longrightarrow S$ such that

$$
\|\xi(\omega)-T(\omega, \xi(\omega))\|=d(T(\omega, \xi(\omega)), S)
$$

for each $\omega \in \Omega$.

Proof. It is clear that $T$ is a 1-set-contractive random operator. We claim that $T$ satisfies the condition $(A)$. Fix $\omega \in \Omega$ arbitrarily. Let $\left\{x_{n}\right\}$ be any sequence in $S$ and $D \in C(S)$ such that $d\left(x_{n}, D\right) \longrightarrow 0$ and $\left\|x_{n}-T\left(\omega, x_{n}\right)\right\| \longrightarrow 0$ (that is, $x_{n}-T\left(\omega, x_{n}\right) \longrightarrow 0$ ) as $n \longrightarrow \infty$. Since $\left\{x_{n}\right\}$ is bounded and $C$ is compact, $C\left(\omega, x_{n}\right) \longrightarrow y$ as $n \longrightarrow \infty$, and hence $x_{n}-B\left(\omega, x_{n}\right)=$ $x_{n}-T\left(\omega, x_{n}\right)+C\left(\omega, x_{n}\right) \longrightarrow y$ or $x_{n}-F\left(\omega, x_{n}\right) \longrightarrow 0$ as $n \longrightarrow \infty$, where $F: \Omega \times S \longrightarrow X$ is a uniformly strictly contractive random operator given by $F(\omega, x)=B(\omega, x)+y$. Since $X$ is reflexive, there exists a subsequence $\left\{x_{m}\right\}$ of $\left\{x_{n}\right\}$ such that $x_{m} \longrightarrow x_{0}$ weakly as $m \longrightarrow \infty$. It follows, from Kirk [6], that $\left\{x_{m}\right\}$ is a Cauchy sequence which necessarily converges strongly to $x_{0}$. The continuity of $T$ further implies that $x_{0} \in D$ and $x_{0}-T\left(\omega, x_{0}\right)=0$. Hence $T$ satisfies the condition $(A)$ and so, by Theorem 3.3 , there exists a measurable map $\xi: \Omega \longrightarrow S$ such that

$$
\|\xi(\omega)-T(\omega, \xi(\omega))\|=d(T(\omega, \xi(\omega)), S)
$$

for each $\omega \in \Omega$. 
Theorem 3.9. Let $S$ be a separable closed ball with center at the origin and radius $r$ in a Banach space $X, T: \Omega \times S \longrightarrow X$ a random operator. Moreover, $T$ satisfies one of the following conditions: (i) $T$ is of strictly semicontractive type; (ii) $T$ is of weakly semicontractive type and satifies the condition $(A)$. Then there exists a measurable map $\xi: \Omega \longrightarrow S$ such that

$$
\|\xi(\omega)-T(\omega, \xi(\omega))\|=d(T(\omega, \xi(\omega)), S)
$$

for each $\omega \in \Omega$.

Proof. If $T$ satisfies (i), then, by Petryshyn[18, Lemma 3.2] $T$ is $k$-set-contractive with $k<1$. To prove the theorem, it suffices to show that $T$ satisfies the condition $(A)$. Fix $\omega \in \Omega$ arbitrarily. Let $\left\{x_{n}\right\}$ be any sequence in $S$ and $D \in C(S)$ such that $d\left(x_{n}, D\right) \longrightarrow 0$ and $\left\|x_{n}-T\left(\omega, x_{n}\right)\right\| \longrightarrow 0$ (equivalently $x_{n}-T\left(\omega, x_{n}\right) \longrightarrow 0$ ) as $n \longrightarrow \infty$. Then, as in the proof of [18, Theorem 3.1$],\left\{x_{n}\right\}$ is precompact and so we may assume that $x_{n} \longrightarrow x_{0}$ in $S$. It further implies that $x_{0} \in D$ and $x_{0}-T\left(\omega, x_{0}\right)=0$. Hence, by Theorem 3.3 , there exists a measurable map $\xi: \Omega \longrightarrow S$ such that

$$
\|\xi(\omega)-T(\omega, \xi(\omega))\|=d(T(\omega, \xi(\omega)), S)
$$

for each $\omega \in \Omega$.

If $T$ satisfies (ii), then, by Petryshyn[18, Lemma 3.2] $T$ is 1-set-contractive. Hence this theorem follows from Theorem 3.3.

Corollary 3.10. Let $S$ be a separable closed ball with center at the origin and radius $r$ in a reflexive Banach space $X$ and $T: \Omega \times S \rightarrow X$ a continuous strongly semicontractive type random operator relative to $X$. Then there exists a measurable map $\xi: \Omega \longrightarrow S$ such that

$$
\|\xi(\omega)-T(\omega, \xi(\omega))\|=d(T(\omega, \xi(\omega)), S)
$$

for each $\omega \in \Omega$.

Proof. Since $T$ is of stongly semicontractive type relative to $X$, it is of semicontractive type. It follows, from Petryshyn[18, Lemma 3.2 and p.338], that $T$ is 1-set-contractive. Moreover, $T$ satisfies the condition $(A)$. Indeed, fix $\omega \in \Omega$ arbitrarily. Let $\left\{x_{n}\right\}$ be any sequence in $S$ and $D \in C(S)$ such that $d\left(x_{n}, D\right) \longrightarrow 0$ and $\left\|x_{n}-T\left(\omega, x_{n}\right)\right\| \longrightarrow 0$ as $n \longrightarrow \infty$. Then, by Kirk[6, Theorem 2], $x_{n} \longrightarrow x_{0}$ in $S$ and so $x_{0} \in D$ with $x_{0}-T\left(\omega, x_{0}\right)=0$. By Theorem 3.3 , there exists a measurable map $\xi: \Omega \longrightarrow S$ such that

$$
\|\xi(\omega)-T(\omega, \xi(\omega))\|=d(T(\omega, \xi(\omega)), S)
$$

for each $\omega \in \Omega$.

Corollary 3.11. Let $S$ be a separable closed ball with center at the origin and radius $r$ in a reflexive Banach space $X, B: \Omega \times S \longrightarrow X$ a continuous LANE random operator, and $C: \Omega \times S \longrightarrow X$ a completely continuous random operator. If $T=B+C$ satisfies the condition $(A)$, then there exists a measurable map $\xi: \Omega \longrightarrow S$ such that

$$
\|\xi(\omega)-T(\omega, \xi(\omega))\|=d(T(\omega, \xi(\omega)), S)
$$


for each $\omega \in \Omega$.

Proof. It follows, from Nussbaum[14], that $T$ is 1-set-contractive. Therefore, the corollary follows from Theorem 3.3.

Using the fact that every continuous hemicompact map satisfies the condition $(A)$, and employing the proof of Theorem 3.3, we have the following random approximation theorem.

Theorem 3.12. Let $S$ be a separable closed ball with center at the origin and radius $r$ in a Banach space $X$ and $T: \Omega \times S \longrightarrow X$ a continuous 1-set-contractive random operator such that the random operator $R T: \Omega \times S \longrightarrow S$ is hemicompact.. Then there exists a measurable map $\xi: \Omega \longrightarrow S$ such that

$$
\|\xi(\omega)-T(\omega, \xi(\omega))\|=d(T(\omega, \xi(\omega)), S)
$$

for each $\omega \in \Omega$.

Remark 3.13. The assumption that $(I-R T(\omega,)).(S)$ is closed for each $\omega \in \Omega$ in Tan and Yuan[24, Theorem 4.1] is superfluous.

\section{Applications to Random Fixed Point Theory}

Adopting the method of Liu[13, Theorem 3.1], we can prove the following theorems and corollaries as applications of our random approximation theorems. We omit their proofs.

Theorem 4.1. Let $S$ be a separable closed ball with center at the origin and radius $r$ in a Banach space $X$ and $T: \Omega \times S \longrightarrow X$ a continuous 1-set-contractive random operator for which the condition $(A)$ holds. Moreover, $T$ satisfies any one of the following conditions:

1. For each $\omega \in \Omega$, each $x \in S_{r}=\{x \in X:\|x\|=r\}$ with $\|T(\omega, x)\|>r$, there exists $y$, depending on $\omega$ and $x$, in $I_{S}(x)=\{x+c(z-x): z \in S, c>0\}$ such that $\|y-T(\omega, x)\|<$ $\|x-T(\omega, x)\|$.

2. $T$ is weakly inward ( that is, for each $\omega \in \Omega, T(\omega, x) \in \overline{I_{S}(x)}$ for $x \in S_{r}$ ).

3. $x \neq \lambda T(\omega, x)$ for each $\omega \in \Omega$ and $x \in S_{r}$ with $\|T(\omega, x)\|>r$ and $0<\lambda<1$.

4. $\|x-T(\omega, x)\| \neq\|T(\omega, x)\|-r$ for each $\omega \in \Omega$ and $x \in S_{r}$ with $\|T(\omega, x)\|>r$.

5. For each $\omega \in \Omega$ and $x \in S_{r}$ with $\|T(\omega, x)\|>r$, there exists $\alpha \in(1, \infty)$ such that $\|T(\omega, x)\|^{\alpha}-r^{\alpha} \leq\|T(\omega, x)-x\|^{\alpha}$.

6. For each $\omega \in \Omega$ and $x \in S_{r}$ with $\|T(\omega, x)\|>r$, there exists $\beta \in(0,1)$ such that $\|T(\omega, x)\|^{\beta}-r^{\beta} \geq\|T(\omega, x)-x\|^{\beta}$.

Then $T$ has a random fixed point.

Corollary 4.2. Let $S$ be a separable closed ball with center at the origin and radius $r$ in a Banach space $X$ and $T: \Omega \times S \longrightarrow X$ a continuous condensing random operator. Moreover, $T$ satisfies any one of the six conditions of Theorem 4.1. Then $T$ has a random fixed point. 
Corollary 4.3. Let $S$ be a separable closed ball with center at the origin and radius $r$ in a Banach space $X, B: \Omega \times S \longrightarrow X$ a random contraction, and $C: \Omega \times S \longrightarrow X$ a continuous compact random operator. If $T=B+C$ satisfies any one of the six conditions of Theorem 4.1, then $T$ has a random fixed point.

Theorem 4.4. Let $S$ be a separable closed ball with center at the origin and radius $r$ in a reflexive Banach space $X, B: \Omega \times S \longrightarrow X$ a uniformly strictly contractive random operator on $S$ relative to $X$, and $C: \Omega \times S \longrightarrow X$ a continuous compact random operator. If $T=B+C$ satisfies any one of the six conditions of Theorem 4.1, then $T$ has a random fixed point

Theorem 4.5. Let $S$ be a separable closed ball with center at the origin and radius $r$ in a Banach space $X$ and $T: \Omega \times S \longrightarrow X$ a continuous random operator of strictly semicontractive type. Moreover, $T$ satisfies any one of the six conditions of Theorem 4.1. Then $T$ has a random fixed point.

Theorem 4.6. Let $S$ be a separable closed ball with center at the origin and radius $r$ in a Banach space $X$ and $T: \Omega \times S \longrightarrow X$ a continuous random operator of weakly semicontractive type for which the condition $(A)$ holds. Moreover, $T$ satisfies any one of the six conditions of Theorem 4.1. Then $T$ has a random fixed point.

Corollary 4.7. Let $S$ be a separable closed ball with center at the origin and radius $r$ in a reflexive Banach space $X$ and $T: \Omega \times S \longrightarrow X$ a strongly semicontractive type random operator relative to $X$. Moreover, $T$ satisfies any one of the six conditions of Theorem 4.1. Then $T$ has a random fixed point.

Corollary 4.8. Let $S$ be a separable closed ball with center at the origin and radius $r$ in a reflexive Banach space $X, B: \Omega \times S \longrightarrow X$ a continuous $L A N E$ random operator, and $C:$ $\Omega \times S \longrightarrow X$ a completely continuous random operator. Moreover, $T=B+C$ satisfies (i) the condition $(A)$ and (ii) any one of the six conditions of Theorem 4.1. Then $T$ has a random fixed point.

Remark 4.9. A careful reading of Theorem 3.12 and Theorem 4.8 of Tan and Yuan [24] reveals that the condition that, for each $\omega \in \Omega,(I-R T(\omega,)).(S)$ is closed in $X$ is redundant.

Acknowledgments. N.S. would like to express his gratitude to the Abdus Salam International Centre for Theoretical Physics, Trieste, Italy, where this work was completed, and the Commission on Development and Exchange of the International Mathematical Union for financial support. 


\section{REFERENCES}

[1] I. Beg and N. Shahzad, Applications of the proximity map to random fixed point theorems in Hilbert space, J. Math. Anal. Appl. 196 (1995), 606-613.

[2] I. Beg and N. Shahzad, Some random approximation theorems with applications, Nonlinear Anal. (1998) (in press).

[3] K. Fan, Extensions of two fixed point theorems of F. E. Browder, Math. Z. 112 (1969), 234-240.

[4] K. Fan, Some properties of convex sets related to fixed point theorems, Math. Ann. 266 (1984), 519-537.

[5] C. W. Ha, Extensions of two fixed point theorems of Ky Fan, Math. Z. 190 (1985), 13-16.

[6] W. A. Kirk, On nonlinear mappings of strongly semicontractive type, J. Math. Anal. Appl. 27 (1969), 409412.

[7] K. Kuratowski and C. Ryll-Nardzewski, A general theorem on selectors, . Bull. Acad. Polon. Sci. Ser. Sci. Math. Astronom. Phys. 13 (1965), 397-403.

[8] T. C. Lin, A note on a theorem of Ky Fan, Canad. J. Math. 22 (1979), 513-515.

[9] T. C. Lin, Approximation theorems and fixed point theorems in cones, Proc. Amer. Math. Soc. 102 (1988), $502-506$.

[10] T. C. Lin, Random approximations and random fixed point theorems for non-self-maps, Proc. Amer. Math. Soc.103 (1988), 1129-1135.

[11] T. C. Lin, Random approximations and random fixed point theorems for continuous 1-set-contractive random maps, Proc. Amer. Math. Soc. 123 (1995), 1167-1176.

[12] T. C. Lin and C. L. Yen, Applications of the proximity map to fixed point theorems in Hilbert space, $J$. Approx. Theory 52 (1988), 141-148.

[13] L. S. Liu, Some random approximations and random fixed point theorems for 1-set-contractive random operators, Proc. Amer. Math. Soc. 125 (1997), 515-521.

[14] R. D. Nussbaum, The fixed point index and fixed point theorems for k-set-contractions, Ph. D. Thesis, University of Chicago 1969.

[15] R. D. Nussbaum The fixed point index for local condensing maps, Ann. Mat. Pura Appl. 89 (1971), 217-258.

[16] N. S. Papageorgiou, Random fixed point theorems for measurable multifunctions in Banach spaces, Proc. Amer. Math. Soc. 97 (1986), 507-514.

[17] N. S. Papageorgiou, Random fixed points and random differential inclusions, Internat. J. Math. \& Math. Sci. 11 (1988), 551-560.

[18] W. V. Petryshyn, Fixed point theorems for various classes of 1-set-contractive and 1-ball-contractive mappings, Trans. Amer. Math. Soc. 182 (1973), 323-352.

[19] V. M. Seghal and S. P.Singh, On random approximations and a random fixed point theorems for set-valued mappings, Proc. Amer. Math. Soc. 97 (1985), 91-94.

[20] V. M. Sehgal and C. Waters, Some random fixed point theorems for condensing operators, Proc. Amer. Math. Soc. 90 (1984), 425-429.

[21] N. Shahzad, The random version of the Kirzbraun-Valentine extension theorem, J. Math. Anal. Appl. 215 (1997), 147-153.

[22] S. P. Singh and B. Watson, Proximity maps and fixed points, J. Approx. Theory 39 (1983), 72-76.

[23] K. K. Tan and X. Z. Yuan, Random fixed point theorems and approximations in cones, J. Math. Anal. Appl. 185 (1994), 378-390.

[24] K. K. Tan and X. Z. Yuan, Random fixed point theorems and approximations, Stoch. Anal. Appl. 15 (1997), 103-123. 